
temps de la convergence?

\title{
Les natifs numériques profitent-ils de la convergence? Constats nuancés et pistes de réflexion pour les éducateurs
}

Do Digital Natives Benefit from Convergence? Contrasted Findings and Implications for Educators

Jacques Kerneis, Alexandre Coutant, Henri Assogba et Thomas Stenger

\section{(2) OpenEdition}

Journals

Édition électronique

URL : http://journals.openedition.org/edc/3386

DOI : 10.4000/edc.3386

ISSN : 2101-0366

Éditeur

Université Lille-3

Édition imprimée

Date de publication : 30 juin 2012

Pagination : 53-68

ISBN : 978-2-917562-07-9

ISSN : $1270-6841$

\section{Référence électronique}

Jacques Kerneis, Alexandre Coutant, Henri Assogba et Thomas Stenger, «Les natifs numériques profitent-ils de la convergence? ? Constats nuancés et pistes de réflexion pour les éducateurs », Études de communication [En ligne], 38 | 2012, mis en ligne le 30 juin 2014, consulté le 01 mai 2019. URL : http://journals.openedition.org/edc/3386 ; DOI : 10.4000/edc.3386

Ce document a été généré automatiquement le 1 mai 2019.

(c) Tous droits réservés 


\title{
Les natifs numériques profitent-ils de la convergence ? Constats nuancés et pistes de réflexion pour les éducateurs
}

\author{
Do Digital Natives Benefit from Convergence? Contrasted Findings and \\ Implications for Educators
}

Jacques Kerneis, Alexandre Coutant, Henri Assogba et Thomas Stenger

1 Les textes de Jenkins consacrés à la culture de la convergence (Jenkins, 2006) manifestent un optimisme, volontiers reconnu par l'auteur, envers ce que pourraient apporter les médias sociaux. On retrouve cet optimisme dans le texte d'Howard Rheingold sur les natifs numériques ${ }^{1}$ (Allard et Blondiaux, 2007), ainsi que dans les propos des promoteurs de ce concept comme Marc Prensky ou du philosophe Michel Serres lorsqu'il évoque les " petites poucettes $»^{2}$. Ces visions, particulièrement présentes dès lors qu'on évoque les médias sociaux, ont en commun de fonder leur optimisme sur une analyse de l'accessibilité offerte par Internet à de nombreux contenus ainsi qu'au potentiel participatif de la technologie sur laquelle il repose ${ }^{3}$.

2 Ces publications ont provoqué de nombreuses polémiques quant à la réalité de leurs apports. Celles-ci n'ont cependant pas empêché que le terme ne se diffuse avec un succès non démenti dans les discours journalistiques ou politiques. Il faut concéder que ces polémiques ont longtemps confronté deux positions radicalement opposées, désormais bien connues sous le nom d'apocalyptiques et d'hyperintégrés (Eco, 1985), mais ayant en commun de se fonder davantage sur des postulats que sur des retours de terrain.

Cette situation évolue depuis quelques temps grâce à la publication d'enquêtes interrogeant les usages et les compétences des jeunes utilisateurs ${ }^{4}$. Cet article propose donc de poser à nouveau la question de la réalité de cette culture de la convergence 
maîtrisée par les natifs numériques à la lumière de ces résultats et de nos propres enquêtes.

4 Dans une première partie, les concepts de culture de la convergence et des natifs numériques sont rappelés et discutés, notamment en mettant en perspective la convergence technique avec celles des contenus et de leurs émetteurs. Les terrains sur lesquels reposent ces réflexions sont ensuite présentés. Une troisième partie se penche plus précisément sur quelques cas d'usages illustrant l'appropriation nuancée des médias sociaux par les jeunes utilisateurs dans le cadre de la sélection et de l'évaluation de l'information, de la gestion de leur identité numérique et de la présence connectée. Une ultime partie conclut sur le rôle renforcé du médiateur pour éduquer à l'usage de ces outils.

\section{Vers une « culture de la convergence »}

5 Il nous paraît utile de clarifier a minima les concepts de culture de la convergence et de natifs numériques. Ces derniers, encore qualifiés de " génération Internet », " génération $Y$ » ou "petite poucette» sont nés et vivent dans un environnement dominé par des technologies de l'information et de la communication individuelles. Certes, il ne s'agit pas de généraliser puisqu'il existe même dans des "pays développés» une proportion non négligeable de jeunes dits « off-line» (Brotcorne et Valenduc, 2009) mais globalement, les natifs numériques sont considérés comme des utilisateurs potentiels des nombreux services qui se créent continûment. Qu'il s'agisse d'espace numérique de travail (ENT), d'outils de partages ciblés ou des mondes virtuels (du type Serious game ou Second Life) ou encore d'outils de mise en réseaux (du type Facebook ou Myspace), les potentialités offertes par les médias sociaux paraissent inépuisables et s'inscrivent dans une dynamique d'évolution permanente rendant chaque fois obsolète le qualificatif «nouveau ». Dans un pareil contexte, les natifs numériques aimeraient apprendre en faisant et considéreraient, à en croire McNelly (2005), l'interaction avec les autres comme essentielle dans le processus d'apprentissage.

6 Ces visions des jeunes utilisateurs rappellent que la confrontation entre des objets d'apprentissage et des outils de communication est l'un des paradoxes que doivent gérer les éducateurs. Une confrontation qui est souvent résolue, comme le mentionne MasselotGirard (1999), par une position quasi immanente, comme si l'usage produisait la connaissance. Or, l'usage n'est pas un objet naturel mais un construit sociotechnique (Perriault, 1992). C'est pourquoi, Moeglin (2005, p. 11), affirme qu'il faut aller au-delà de la «naturalité apparente » des « technologies éducatives ». En effet, les outils et médias ne deviennent éducatifs que s'il y a une « aptitude qui leur est reconnue de permettre à des élèves d'apprendre et à des maîtres d'enseigner, de s'informer et de communiquer en contexte éducatif». Pour Moeglin (2005, p. 245), "l'intégration technique à laquelle concourent multimédia et convergence peut donner l'illusion de l'avènement d'un paradigme numérique unitaire » mais il n'en est rien, conclut-il.

7 Par convergence, Henry Jenkins (2006) désigne la circulation croissante des contenus entre médias et le fait que les utilisateurs, dans ce contexte, se portent plus volontiers au devant de ces contenus, pour éventuellement se les approprier. La convergence au sens de Jenkins, c'est donc avant tout ce que les acteurs font des technologies et moins les technologies elles-mêmes. Pour cet auteur, la convergence représente un changement culturel dans la mesure où les consommateurs sont encouragés à rechercher, par leurs 
propres moyens, de nouvelles informations et à connecter entre eux des contenus médiatiques disparates. Si on peut se réjouir d'une responsabilisation accrue de l'utilisateur, la convergence ainsi comprise suppose et exige de la part de ce dernier la maîtrise de compétences supplémentaires de distinction des contenus et de leurs auteurs. C'est sur ce point que la notion a provoqué de nombreuses polémiques. En s'en tenant à quelques exemples heureux, ses promoteurs occultent les nombreux cas où celle-ci ne représente un gain pour l'utilisateur. À titre d'illustration, on peut évoquer les sites d'informations où le travail du journaliste et les commentaires des lecteurs se trouvent réunis sur la même page, ou ceux ne mettant pas en avant la distinction entre les articles des journalistes et ceux des abonnés ${ }^{5}$. Il en va de même des nombreux blogs proposant les analyses de la part de rédacteurs aux profils et motivations hétéroclites, ce qui impose de savoir distinguer la crédibilité à accorder à chacun. Or, ce type de compétence demeure très inégalement réparti dans la population (Rebillard, 2007). Les médias classiques jouaient ce rôle de sélection et c'est l'enjeu de la difficile mise en place d'une éducation aux médias que de tenter de diffuser ces compétences (Jacquinot, 2001).

8 Cet article se propose d'analyser des cas concrets de ces enjeux de connaissance supplémentaires qu'engendre la convergence auprès des natifs numériques afin de fournir une vision plus réaliste des bénéfices qu'ils en tirent ou des difficultés auxquels ils font face.

\section{Deux terrains complémentaires : précisions méthodologiques}

9 Ces réflexions sont issues des travaux entourant un projet de recherche et un projet pédagogique filmé, menés auprès de jeunes utilisateurs allant du collège à l'entrée dans la vie active.

10 Le premier recueil de matériau a été effectué dans le cadre d'une enquête portant sur les activités des jeunes utilisateurs sur les réseaux socionumériques (Rsn). L'enquête, se déroulant sur deux ans, a successivement travaillé à la définition précise de ces plateformes au sein de la nébuleuse "médias sociaux», aux manières de s'inscrire et de s'approprier le dispositif sociotechnique propre à ces sites, à la compréhension des activités très spécifiques qu'y mènent les jeunes utilisateurs, à la mise à jour des enjeux de présentation de soi auxquels ces activités répondent, à la caractérisation des liens sociaux noués dans ce contexte sociotechnique particulier ainsi qu'aux formes d'influence qui s'exercent entre les différents acteurs retrouvés sur les Rsn. Elle a reposé sur une méthodologie plurielle cumulant 24mois d'observation participante sur les principales plateformes (Facebook, Skyrock, Myspace), entretiens individuels et de groupes ( $\mathrm{n}=65)$, analyse systématique de profils $(n=38)$, questionnaire sur les principaux usages $(n=635)$ et analyse quantitative de 7041profils menée à l'aide du logiciel de traitement de données Tetralogie ${ }^{6}$.

11 La question de l'appropriation des Rsn a constitué l'occasion d'aborder les formes d'apprentissage mises en œuvre par les jeunes utilisateurs ainsi que les compétences qu'ils mobilisaient, se devaient d'acquérir ou dont ils manquaient. Notamment, les questions des fréquence et lieux de connexion ont permis de soulever les conflits provoqués par la facilité d'accès à ces plateformes dans les contextes éducatifs et familiaux. La discussion s'est alors régulièrement poursuivie sur ce qu'apportaient les Rsn 
comme connaissances, prises ici au sens large, celles qu'ils n'apportaient pas, voir celles dont ils détournaient en incitant à leur usage. Le lien entre les déclaratifs des enquêtés et l'observation de leurs pratiques sur les sites a permis d'approfondir la compréhension de ces enjeux.

Le second corpus a l'originalité de ne pas avoir été constitué spécifiquement pour une enquête scientifique. Il s'agit de débats animés par des étudiants de deuxième année de licence administration économique et sociale (AES) dans le cadre d'un cours de méthodologie de l'oral. Les sujets des débats, librement choisis, ont régulièrement porté sur les Rsn. Trois débats ont abordé d'une manière ou d'une autre l'usage de Facebook. Le premier portait sur l'influence des médias sociaux d'une manière générale. Le second a envisagé ce site sous plusieurs aspects, quand le dernier s'est centré sur ses « dangers». Chaque débat a réuni des étudiants différents et chacun des 5 intervenants au débat (un animateur accompagné de quatre protagonistes) était observé par un autre étudiant. Chacun s'est ensuite exprimé sur les prises de position de celui ou celle qu'il observait et des échanges ont eu lieu pour préciser dans quelle mesure les propos tenus reflétaient leur propre opinion. Ce contexte de recueil particulier doit être interrogé dans la mesure où des rôles contrastés étaient attendus et qu'un cours sur les différents genres de l'oral en général et des débats en particulier avait été donné précédemment aux étudiants. Cependant, la participation se faisait sur la base du volontariat.

13 Ce corpus permet de mettre en évidence les aspects des Rsn qui semblent problématiques. La focalisation des débats sur Facebook est révélatrice de l'importance qu'il revêt aux yeux des jeunes. À de nombreuses reprises, il est question de la nécessité d'un apprentissage des nouveaux médias et les étudiants admettent assez facilement qu'ils «naviguent à vue».

\section{Les natifs numériques profitent-ils de la convergence?}

14 Afin de discuter les théories de la convergence et des digital natives, nous allons à présent décrire trois cas de pratiques de jeunes observées sur les médias sociaux. Ils portent sur la sélection et l'évaluation de l'information consommée, sur la répartition temporelle des contextes et la compartimentation des dimensions de la vie quotidienne et sur la compréhension et la maîtrise de «quoi révéler » selon les configurations sociotechniques sur lesquelles ils se trouvent.

\section{Les jeunes face à la sélection et l'évaluation de l'information}

Les étudiants interrogés évoquent des transformations profondes apportées par les TIC récentes. Nos deux corpus se répondent ainsi de manière inattendue. En effet, les étudiants parlent souvent des plus jeunes (10-18ans) pour aborder, "en creux», la question des nécessaires apprentissages, avec la ferme conviction qu'ils s'en démarquent dans leur rapport aux TIC. Les observations menées dans le cadre du premier terrain permettent cependant de constater qu'en ce qui concerne les cas d'usages évoqués ici, les enquêtés les plus âgés peuvent témoigner des mêmes pratiques que les plus jeunes. Soulignons ce biais souvent rencontré en entretien où les enquêtés projettent sur des 
populations qu'ils estiment plus faibles les risques ou pratiques peu légitimes que l'on retrouve aussi chez eux.

16 Une étudiante souligne : "tout est maintenant accessible, ils ne font pas attention, ils n'éprouvent pas le besoin d'aller chercher plus loin ». Ils sont nombreux à considérer que cette abondance est mal utilisée par les plus jeunes : « tout ce qui est sur Internet est bon à prendre ». Ils évoquent pourtant, si le sujet est lancé, le caractère problématique d'une encyclopédie de type Wikipedia. Ils sont donc capables d'envisager l'importance de la question de la fiabilité de l'information. Malgré tout, cela ne se traduit pas par une adaptation de leurs procédures de recherche à ce contexte. Notamment, les premières pages renvoyées par Google demeurent l'unique terrain d'investigation de tous les étudiants interrogés ${ }^{7}$. Les résultats quantitatifs tout comme les discussions sur les différents usages d'Internet provoquées en entretien lors du premier terrain mettent d'ailleurs en lumière une catégorisation des sites par usage de la part des étudiants qui laisse la part belle aux sites de sociabilité ou d'achats. La recherche d'informations, cantonnée essentiellement à Google et Wikipedia, demeure une activité mineure (Coutant et Stenger, 2009). Quinio aboutit au même constat à propos des étudiants entrant dans une grande école de commerce parisienne à la rentrée 2010 : leur usage d'Internet reste essentiellement à vocation de loisir et de sociabilité et ils témoignent de compétences très limitées en matière de recherche d'information ${ }^{8}$. Ajoutons que la notion de convergence ou les pratiques auxquelles elle renvoie sont peu illustrées dans les échanges. Si quelques étudiants font état de pratiques pervasives, celles-ci demeurent plutôt l'exception au sein d'usages majoritairement cantonnés à un site ou terminal (le badinage pour Facebook, Wikipedia pour la connaissance, le SMS pour les communications avec les intimes, etc).

17 L'accroissement exponentiel du nombre de ressources ne s'accompagne donc pas mécaniquement de compétences nouvelles concernant la sélection de l'information et plus largement de son évaluation. Si l'accessibilité à de nombreux contenus est acquise pour les internautes, dans les pays industrialisés tout du moins, leur intelligibilité suppose plus que jamais l'accumulation de capitaux culturels ne relevant pas d'une culture technique mais bien d'une culture de l'information. Par ailleurs, la profusion et l'accessibilité des contenus ne viennent pas nécessairement encourager cette dernière tant les apprentissages à effectuer se trouvent mis en concurrence avec des pratiques de sociabilités bien plus attrayantes aux yeux des enquêtés. Souligner cette nécessité d'acquérir des compétences dépassant la maîtrise technique ne revient en rien à nier la capacité des internautes à s'en doter. Des enquêtes portant sur les consommateurs de contenus informatifs en ligne dans leur ensemble démontrent d'ailleurs la pluralité de leur consommation. L'idée de convergence se trouve cependant à nouveau mise en cause dans ce cas, mais pour d'autres raisons : «Internet comme hypermédia doit - sur un plan sociologique- moins être évalué en termes de convergence des acteurs, des temporalités et des contenus sur un seul réseau de diffusion, qu'en termes d'élargissement des modes de production et de décloisonnement des conditions de réception des produits médiatiques en ligne » (Rouquette, 2010, p. 6).

\section{La question de l'accessibilité de ces plateformes en toute circonstance}

Un deuxième point de réflexion concerne la gestion des différentes dimensions de la vie sociale lorsque ces technologies deviennent accessibles en permanence. De la sorte, il 
devient d'autant plus nécessaire pour les jeunes utilisateurs de savoir orchestrer leur emploi du temps pour conserver un espace à la famille, aux études, aux activités extrascolaires, etc. Ici aussi, le constat est nuancé. Si aucun cas extrême de dépendance n'est recensé, force est de constater que cette gestion de la déconnexion ne va pas de soi. Les plus jeunes reconnaissent les régulières tensions familiales dues à leur volonté de rester connectés en permanence, ce qui les conduit à souvent être absents cognitivement. Les plus âgés admettent pour leur part ne plus suivre les cours à la moindre perte de rythme. Plus encore, ils disent conserver en permanence leurs outils de communication préférés ouverts, Facebook en tête, ce qui peut amener à les déconcentrer même lorsqu'ils sont décidés à rester assidus. Tous soulignent la difficulté à s'extraire du flux permanent de nouveaux contenus, allant jusqu'à se qualifier d'«addicts». Cette peur de rater le moindre événement sur des sites pourtant reconnus par eux-mêmes comme consacrés à des badineries illustre bien la confusion que provoquent les mémoires numériques entre mémorisable et mémorable (Hoog, 2009). Bien entendu, les études risquent d'en pâtir. Pourtant, s'ils admettent que la pratique assidue des Rsn peut amener une diminution de la lecture et du travail scolaire, les enquêtés refusent bien souvent d'y voir un lien direct. Selon eux, il s'agit plutôt d'un « manque de motivation », intrinsèque à ces activités moins ludiques. Cet aspect est effectivement à prendre en compte dans la réflexion. Les usages scolaires sont confrontés à d'autres usages plus ludiques et "sociaux", dans une concurrence pour l'attention qui apparaît déloyale. Il s'agit alors de développer la capacité d'envisager les bénéfices à long terme du renoncement à des activités plus agréables dans le hic et nunc, ce qui s'avère complexifié par le flot incessant de nouvelles sollicitations.

19 Face à l'omniprésence des outils leur permettant de se connecter à leurs sites préférés, on constate un décalage entre leurs usages catégorisés des différents services proposés sur Internet et leur manière d'envisager des solutions aux tensions que leur connexion permanente provoque. Nous l'avons évoqué supra, les enquêtés sont tout à fait capables de classer les sites selon les usages qu'ils en font. En revanche, dès lors qu'ils sont interrogés sur les solutions envisageables pour conserver un équilibre entre les dimensions de leur vie en ligne et hors ligne, ils ne répondent que par des solutions radicales et appliquées à Internet pris dans sa globalité - "quitter Internet", "se déconnecter " par exemple. Ici, encore, nous sommes bien face à un manque de repères qui seraient utiles pour produire des distinctions dans ce «tout». En leur absence, les solutions envisagées demeurent peu plausibles d'être appliquées car impliquant trop de renoncements.

\section{Une gestion problématique de l'identité numérique : de nouvelles compétences}

20 Les deux terrains soulèvent systématiquement un paradoxe concernant la vie privée : si les enquêtés se déclarent concernés par le sujet, les discussions laissent vite apparaître que cela ne se traduit pas par une mobilisation pour contrôler ses identités numériques. Ce désintérêt transparaît à plusieurs niveaux :

- Par un discours projectif à l'égard des plus jeunes, du type «ils ne savent pas que...", rapidement suivi d'un aveu d'incompétence si l'on demande quelles précautions ils ont pris de leur côté ${ }^{9}$; 
- Par une résignation pessimiste envers un big brother présumé capable de tout obtenir d'eux ;

- Par la remise en cause de l'intérêt des traces ainsi collectées qui évite de se poser la question de la pérennité de celles-ci et leur circulation devant de multiples audiences ;

- Par l'évocation des marketeurs comme principale audience non souhaitée. Les plus âgés incluent aussi les recruteurs et les plus jeunes les prédateurs mais jamais les proches avec lesquels on ne voudrait pas partager certaines choses ou les «ex" proches, pourtant la cause de la plupart des complications, graves ou moins graves, observées en ligne. Ajoutons, concernant les plus jeunes, le peu de conscience des éléments permettant à un éventuel prédateur de les retrouver puisqu'ils estiment être protégés en ne révélant pas leur nom et leur adresse, mais partagent leur collège, les lieux de leurs activités extrascolaires ou des parties de leur emploi du temps ;

- Par la visibilité effective des profils ou de parties importantes de ceux-ci malgré l'absence de lien direct, et parfois même indirect, lors des observations en ligne.

21 Ici aussi, bien que les jeunes développent des usages différents selon les sites qu'ils fréquentent, leurs réponses témoignent de leur incapacité à aborder les enjeux de vie privée autrement que comme un tout. Pourtant, ces enjeux ne se posent pas du tout de la même manière sur Facebook, Twitter, Viadeo ou Skyrock. Les données partagées sont différentes, leur sensibilité aussi, tout autant que les audiences circulant sur ces sites.

Ils en arrivent ainsi très vite à la question radicale et insoluble «faut-il quitter Internet ? ", dont l'observation prouve à quel point elle ne permet pas de résoudre quoi que ce soit: il n'est pas du tout nécessaire d'avoir créé un compte pour apparaître sur Internet, à travers les productions des autres (Coutant et Stenger, 2011).

Pour autant, il serait erroné d'en conclure leur soumission à l'ordre logiciel ainsi imposé par Internet. Bien au contraire, ces incompétences relèvent d'un désintérêt pour ces questions, bien compréhensible une fois le contexte de la plupart de leurs pratiques rappelé. En effet, nous avons souligné à plusieurs reprises que celles-ci concernent essentiellement la sociabilité. Or, il est rare que ces contextes entre pairs nécessitent un apprentissage formel. Il devient donc difficile d'exiger d'eux d'apprendre des règles de présentation de soi proposées de l'extérieur dans un espace qu'ils conçoivent comme uniquement générationnel et précisément construit en opposition avec les normes des générations précédentes.

La différence se fait d'ailleurs ressentir dès qu'Internet commence à être envisagé comme un lieu d'échanges professionnels. Les étudiants les plus proches du marché du travail témoignent alors d'une attitude différente et commencent soit à apprendre à gérer leur visibilité en ligne à l'aide des outils fournis par les plateformes, soit à mettre en place des braconnages plus ou moins élaborés (double compte sur la même plateforme, pseudonymat, mise en visibilité des comptes « officiels » de certains sites, effacement de certains contenus).

\section{Des pistes pour les éducateurs}

Ces exemples démontrent qu'il ne suffit pas d'être né dans le contexte du développement de la convergence pour en profiter, mais qu'il s'agit bien de construire une culture de la convergence. Les éducateurs (enseignants, parents...) jouent toujours ici un rôle déterminant de médiateurs (Merzeau, 2010), même si cette acculturation peut passer par 
d'autres chemins. S'interroger sur l'éducation aux médias sociaux ou par les médias sociaux demeure cependant une interrogation trop générale pour l'éducateur cherchant à en tirer parti ${ }^{10}$. Il lui est nécessaire de pouvoir distinguer les sites qui les composent selon le potentiel éducatif qu'ils recèlent et les compétences qu'ils requièrent afin de pouvoir transmettre cette vision diversifiée des services trouvés sur Internet. La matrice proposée par Schugurensky (2007) constitue un outil pour identifier les espaces où les utilisateurs pourront témoigner d'un intérêt face à la mise en place d'éléments pédagogiques ou d'un intérêt à apprendre à s'y comporter :

FIgURE 1 : LES FORMES D'APPRENTISSAgE INFORMEL DÉfiNIES PAR SCHUgURENSKY

\begin{tabular}{|l|l|l|}
\hline APPRENTISSAGES & Intentionnels & Conscients \\
\hline Auto-dirigés & Oui & Oui \\
\hline Fortuits & Non & Oui \\
\hline Socialisation & Non & Non \\
\hline
\end{tabular}

Les sites où l'on constate des apprentissages auto-dirigés constituent le lieu le plus propice à la mise en place de démarches pédagogiques (exemples: blogs ou forums s'intéressant à un sujet particulier et réunissant des amateurs ou passionnés, wikis...). Elles peuvent aussi se développer sur les sites qui permettent des apprentissages fortuits, bien que le contexte non intentionnel de ces apprentissages implique de développer ce que l'économie de l'attention nomme le «style» (Kessous, Mellet et Zouinar, 2010) pour s'assurer de l'intérêt des personnes visées (exemples : sites de réseautage professionnel, blogs et communautés davantage tournés vers la sociabilité mais regroupant les utilisateurs par centres d'intérêt). Enfin, le contexte de socialisation, s'il permet des formes d'apprentissage superficielles ${ }^{11}$ et de découvrir les normes de l'interaction propres à sa culture générationnelle (Allard et Blondiaux, 2007), place les utilisateurs dans un contexte où ils risquent de ne pas se rendre disponibles pour des contenus pédagogiques (exemples : réseaux socionumériques ou univers virtuels de socialisation). Ces contextes de socialisation en ligne peuvent en revanche nécessiter une éducation à leur utilisation, mais qu'il est difficile d'entreprendre sur les sites eux-mêmes, qui proposent des activités badines intéressant bien davantage les utilisateurs (Stenger et Coutant, 2011).

Les différentes expérimentations heureuses menées à l'école ${ }^{12}$ autour de Twitter amènent aux mêmes conclusions : « le travail consiste à intégrer ces outils pour servir des finalités classiques de l'éducation : l'utilisation de Twitter en classe donne du sens aux activités de production d'écrit. Twitter joue pleinement son rôle de motivation: les élèves comprennent pourquoi ils écrivent et s'investissent plus facilement dans les apprentissages. Cependant, beaucoup de pratiques restent à inventer, afin d'explorer d'autres pistes d'apprentissage ». Dans ces pratiques, insistons sur les relations parents/ école ou le lien élève/école hors les murs (en stage par exemple) qui constituent des terrains sur lesquels on peut construire des projets pédagogiques et communicationnels.

Twitter offre en outre l'avantage d'initier à la gestion des plateformes fonctionnant sur le modèle du flux. Les problématiques d'archivage, de tri de l'information, de distinction entre mémorisable et mémorable évoquées supra peuvent donc être abordées, sans avoir 
à passer par Facebook dont la finalité de sociabilité rend les démarches pédagogiques difficiles à mettre en place. Une enseignante de terminale souligne: «Utiliser Twitter permet ainsi d'entrer sur le terrain encore vierge d'un réseau social. Cette initiation pose les règles sans interférences. Les élèves utilisent beaucoup le net et Facebook mais sans jamais y avoir été éduqués. Les initier à un réseau social inconnu, c'est aussi les éduquer à un usage citoyen d'un réseau social : mettre en place des règles, à construire avec eux une réflexion sur leur identité numérique. Au fur et à mesure de cette éducation civique du net, j'ai vu leurs profils Facebook se modifier, les élèves apprendre à mieux se protéger ou au moins à réfléchir aux informations qu'ils diffusent. Éduquer les élèves à Twitter c'est apprendre aux élèves à réfléchir à leurs usages de Facebook $»^{13}$.

Reste enfin à aborder l'inédite question de l'accessibilité de ces médias sociaux qui, couplée à leur mise à jour permanente, favorise un envahissement des autres espaces/ temps en privilégiant les contacts et les signes de présence récents de chacun. Nul doute qu'un regard distancié sur cette "pratique de flux » serait utile. Les expériences relatées ici montrent que cet aspect constitue encore l'enjeu pédagogique le plus complexe à gérer : «À ce stade de l'année, la plus grande difficulté est la gestion du temps : l'activité Twitter est prioritaire sur toutes les autres $»^{14}$.

Cette convergence médiatique offre bien un potentiel de parcours d'apprentissage intéressants, qu'ils soient institutionnels ou non. Pour les faire se réaliser, il nous semble plus perspicace d'inventer, à petits pas, des manières d'employer ces médias sociaux au sein d'une translittératie (Thomas, 2007), que de décréter une nécessaire révolution du rôle de l'enseignant. Rappelons en effet que les programmes éducatifs n'évoluent pas à la même vitesse que les technologies. Cela signifie qu'il est nécessaire de mettre en place une formation des éducateurs de qualité, afin qu'ils sachent eux-mêmes distinguer les sites et les exploiter au mieux.

\section{BIBLIOGRAPHIE}

Allard L. et Blondiau O. (dir.), (2007), 2.0 ? Culture Numérique, Cultures Expressives, in Médiamorphoses, $\mathrm{n}^{\circ} 21$, pp. 7-124.

Boyd D., (2007), Information Access in a Networked World, http://www.danah.org/papers/talks/ Pearson2007.html, date de la dernière visite : 10 septembre 2011.

Brotcorne P. et Valentuc G., (2009) « Les compétences numériques et les inégalités dans les usages d'internet ", in Les Cahiers du Numérique, vol. $5, n^{\circ} 1$, Hermès Lavoisier.

Coutant A. et Stenger T., (2009), Les configurations sociotechniques sur le Web et leurs usages : le cas des réseaux socionumériques, 7eColloque du Chapitre Français de l'ISKO : Intelligence Collective et Organisation des Connaissances, 25-26 juin, Lyon (France), pp. 27-34.

Coutant A. et Stenger T., (2010), Pratiques et temporalités des réseaux socionumériques : logique de flux et logique d'archive, Médiation et Information, $\mathrm{n}^{\circ}$ 32, 2010, pp. 125-136. 
Coutant A. et Stenger T., (2011), Production et gestion d'attributs identitaires, Les Cahiers du Numérique, vol. 7, $\mathrm{n}^{\circ}$ 1, pp. 61-74.

Dewey J., (1938/1967), Logique, la théorie de l'enquête, Paris, Presses Universitaires de France, 693 p.

Eco U., (1985), La guerre du faux, Paris, Grasset.

Hoog E., (2009), Mémoire, Année zéro, Paris, Seuil.

Jacquinot G. (dir.), (2001), Les jeunes et les médias, Perspectives de la recherche dans le monde, Paris, L'Harmattan.

Jenkins H., (2006), Convergence culture: where old and new media collide, New-York, NY University Press.

Kessous E., Mellet K. et Zouinar M., (2010), L'Économie de l'attention : Entre protection des ressources cognitives et extraction de la valeur, Sociologie du travail, $n^{\circ} 3$, pp. 359-373.

Le Deuff O., (2011), Éducation et réseaux socionumériques : des environnements qui nécessitent une formation, Hermès, $n^{\circ}$ 59, pp. 67-74.

Masselot-Girard M., (1999), Lire, interpréter, évaluer : des lecteurs-acteurs, Actes du colloque Multimédia et construction des savoirs, Besançon, 25-28mai1999, pp. 7-13.

Mc Nelly B., (2005), Using technology as a learning tool, not just a cool new thing, in D. Oblinger \& J. Oblinger (Éds.), Educating the Net Generation, 4.1-4.10, Boulder, Educause2005.

Merzeau L. (dir.), (2010), Présence : de la gestion d'une identité à l'exercice d'une liberté, Documentaliste, vol. $47, \mathrm{n}^{\circ} 1$.

Moeglin P., (2010), Une approche transdisciplinaire des outils et médias éducatifs, Actes du Congrès de la SFSIC, http://tabarqa.u-bourgogne.fr/outils/OconfS/index.php/SIC/SFSIC17/paper/view/144, date de la dernière visite le : 10 septembre 2011.

Moeglin P., (2005), Outils et médias éducatifs : une approche communicationnelle, Grenoble, Presse universitaires de Grenoble.

Perriault J., (1992), La logique de l'usage, Paris, Flammarion.

Rebillard F., (2007), Le Web2.0 en perspective. Une analyse socio-économique de l'Internet, Paris, L'Harmattan.

Rheingold H., (2007), Natifs numériques ou des raisons d'être optimistes, Médiamorphes, $\mathrm{n}^{\circ} 21$, pp. 28-30.

Rouquette S., (2010), Convergence ou diversification des pratiques de consommation des informations médiatiques en ligne ? Actes du 17econgrès de laSFSIC, http://tabarqa.ubourgogne.fr/outils/OconfS/index.php/SIC/SFSIC17/paper/view/12, date de la dernière visite le : 10 septembre 2011.

Schugurensky D., (2007), Vingt mille lieues sous les mers : les quatre défis de l'apprentissage informel, Revue française de pédagogie, $n^{\circ} 160, \mathrm{pp} .13-27$.

Stenger T. et Coutant A., (2011), Les réseaux numériques dits sociaux, Hermes, $\mathrm{n}^{\circ} 59$.

Tchuente D., Canut M.-F., Baptiste-Jessel N., Coutant A., Stenger T. et Rampnoux O., (2011), Pour une approche interdisciplinaire des TIC : le cas des réseaux socionumériques, Document Numérique, vol. 14, $\mathrm{n}^{\circ} 1$, pp. 31-57. 


\section{NOTES}

1. Traduction de « digital natives ».

2. http://www.liberation.fr/culture/01012357658-petite-poucette-la-generation-mutante.

3. Rheingold qualifiera d'ailleurs Jenkins de « Mac Luhan du XXI ${ }^{\mathrm{e}}$ siècle » à l'occasion de la sortie de Convergence Culture. Heureux rapprochement en ce qui concerne notre article puisqu'il s'agit de discuter l'importance donnée aux supports aux dépens des usages de ces derniers dans les théories de Jenkins, dans le prolongement des discussions ayant fait suite aux publications des thèses de Mac Luhan.

4. Par exemple : http://www.agent4change.net/resources/research/1088.

5. Voir, par exemple, le format de Mediapart qui se définit comme « un journal d'information numérique, indépendant et participatif » où les articles des blogs des abonnés apparaissent au milieu des articles des membres de la rédaction.

6. Projet de recherche «RSn: Réseaux socionumériques » financé par La Poste (Direction de l'Innovation et des E-services - DIDES - et Mission Recherche et Prospective), coordonné par Thomas Stenger et réalisé par une équipe de sept chercheurs des laboratoires CEREGE et IRIT.

7. Ajoutons que la recherche d'informations peut même être très facilement évitée par certains.

8. Enquête évoquée lors du séminaire Identités Numériques du 28 juin 2011, à paraître : http:// www.iscc.cnrs.fr/spip.php?article1383.

9. Une récente enquête réalisée pour la Commission européenne appuie ces remarques en signalant que l'âge des enfants utilisant les Rsn diminue et que beaucoup ignorent les risques qu'ils prennent par rapport à leur vie privée : http://europa.eu/rapid/pressReleasesAction.do? reference $=I P / 11 / 479 \&$ format $=$ HTML\&aged $=0$ \&language=FR\&guiLanguage $=e n$.

10. L'expérience relatée sur le blog «Ma dixième année » souligne bien que les enseignants doivent être formés pour savoir distinguer les potentiels des sites. L'auteure y explique en détail la réflexion qui l'a amenée à sélectionner Twitter : http://frompennylane.blogspace.fr/2299814/ Bilan-d-une-annee-d-experimentation-de-twitter-en-classe/.

11. Comme l'apprentissage «par osmose » (Boyd, 2007), initié par le simple fait de se trouver en contact avec des pratiques ou des informations, sans même s'y intéresser ou avoir conscience d'un apprentissage.

12. http://amandineter.free.fr/Journal_Twitter_Cycle_3/Journal/Journal.html, http:// owni.fr/2011/07/07/vous-\%C2\%AB-tweetez-\%C2\%BB-\%E2\%80\%A6-eh-bien-lisez-maintenant/.

13. http://frompennylane.blogspace.fr/2299814/Bilan-d-une-annee-d-experimentation-detwitter-en-classe/. Un enseignant de primaire en arrive aux mêmes conclusions: "Utiliser Twitter dès le CP, ce n'est pas seulement apprendre à écrire, c'est apprendre à écrire pour être lu. Assez vite dans l'année, des points importants ont été dégagés, concernant la politesse, le respect de la vie privée, la loi ou la sécurité. Nous avons élaboré un 'code de Twitter', qui à l'image du code de la route, donne quelques règles quant à son utilisation ».

14. http://owni.fr/2011/07/07/vous-\%C2\%AB-tweetez-\%C2\%BB-\%E2\%80\%A6-eh-bien-lisezmaintenant/. 


\section{RÉSUMÉS}

L'enthousiasme ou la défiance vis-à-vis de la culture de la convergence et des natifs numériques peuvent aujourd'hui être discutés à la lumière de recherches sur les pratiques effectives des utilisateurs. Cet article fondé sur deux terrains complémentaires (un projet scientifique et un projet pédagogique) menés par deux équipes de recherche, examine l'usage des médias sociaux chez de jeunes français. En étudiant trois enjeux centraux: l'évaluation de l'information, le management de son identité numérique et le temps de connexion, l'analyse révèle une certaine facilité d'accès mais aussi de plus grands défis en termes de compétences. Des pistes de réflexion pour une éducation aux médias sociaux sont esquissées dans la dernière partie.

Enthusiasm for or distrust of a convergence culture and of digital natives can now be discussed in light of research on users' actual practices. This paper, which is based on two complementary field investigations (a research project and an educational project) led by two research teams examines the uses of social media by young French people. Three major issues are considered: a critical evaluation of information, digital identity management, and the duration of connections. The analysis reveals clear ease of access but also significant challenges for users in terms of skills. Some thoughts on educational approaches to social media are outlined.

\section{INDEX}

Keywords : social media, convergence, digital native, identity, skills

Mots-clés : médias sociaux, convergence, natif numérique, identité, compétence

\section{AUTEURS}

JACQUES KERNEIS

Jacques Kerneis - Membre associé au CREAD - IUFM de Bretagne, Université de Bretagne Occidentale

\section{ALEXANDRE COUTANT}

Alexandre Coutant - Membre d'ELLIADD, équipe OUN - Université de Franche Comté

HENRI ASSOGBA

Henri Assogba - Membre de la chaire de journalisme scientifique Bell Globemedia - Université Laval du Québec

THOMAS STENGER

Thomas Stenger - Membre du CEREGE - IAE, CEPE, Université de Poitiers 